\title{
Análisis de la labor docente desde la perspectiva de la responsabilidad social interna
}

\author{
Analysis of the work of teachers from the perspective \\ of internal social responsibility
}

ISSN 1510-2432 - ISSN 1688-9304 (en línea) - DOI: https://doi.org/10.18861/cied.2016.7.2.2608

\begin{abstract}
Ricardo Gaete Quezada
Doctor en Procesos de Cambio Social, Universidad de Valladolid España, Magíster en Gestión Pública, Universidad Complutense de Madrid España. Docente e Investigador, Departamento de Ciencias Sociales, Universidad de Antofagasta Chile.
\end{abstract}

Fabián Henríquez

Magíster en Ciencias Sociales Mención Gestión Directiva, Universidad de Antofagasta Chile. Profesor de Historia y Geografía Universidad del Mar y Liceo Científico Humanista La Chimba B-36 Antofagasta, Chile.

Fecha de recibido: 09/10/2015

Fecha de aceptado: 05/09/2016

\section{Resumen}

En el artículo se analizan los principales resultados obtenidos en el estudio desarrollado para comprender la labor de los docentes de aula de un establecimiento de educación municipal chileno desde la perspectiva de los principales lineamientos de la responsabilidad social interna. Fue realizado mediante una investigación con enfoque cualitativo considerando como método de investigación el estudio de caso de carácter único e intrínseco, la entrevista semi-estructurada como técnica de recolección de datos y los procedimientos de la teoría fundamentada como medio para identificar las categorías, conceptos y códigos relacionados con la labor docente. Los resultados obtenidos evidencian importantes problemas de conciliación entre trabajo y familia, producto de las extensas jornadas de trabajo que en ocasiones han desembocado en conflictos familiares, situación agravada por condiciones de trabajo inadecuadas, especialmente en cuanto a la prevención de riesgos en el establecimiento escolar y al bajo nivel de las remuneraciones percibidas por los profesores.

Palabras clave: responsabilidad social, recursos humanos, educación pública, docentes, conciliación trabajo-familia, remuneraciones, prevención de accidentes.

\begin{abstract}
The article analyzes the main findings of the study carried out with the aim of understanding the work of classroom teachers in a Chilean municipal education facility, from the perspective of internal social responsibility guidelines. The analysis was conducted through a qualitative approach including as research method a unique and intrinsic case study, and a semi-structured interview as data collection technique and procedures of grounded theory to identify categories, concepts and codes relating to teaching tasks. The results obtained show significant problems of reconciling work and family issues, derived from long working hours which at times have led to family conflicts, a situation aggravated by inadequate working conditions especially in terms of risk prevention at school facilities and the low level of remuneration received by teachers.
\end{abstract}


Keywords: social responsibility, human resources, public education, teachers, work-family relationship, remuneration, accident prevention.

\section{Introducción}

El presente artículo expone los principales resultados de la investigación que analiza la labor del docente de aula de un establecimiento educacional perteneciente a la Corporación Municipal de Desarrollo Social (CMDS) de la Municipalidad de Antofagasta en Chile, considerando los planteamientos y perspectivas de la responsabilidad social interna (RSI).

En términos generales, la RSI plantea que las organizaciones laborales deben considerar como parte interesada muy relevante a las personas que desempeñan funciones y tareas en los diferentes puestos de trabajo que componen su estructura organizacional (Patrus et al., 2013; Saldarriaga, 2013; Díaz y Lima, 2015; Mei, 2016). Así, la RSI se vincula fuertemente con la aplicación de un nuevo paradigma en la gestión de recursos humanos considerando aspectos tan importantes como la gestión de la diversidad, la conciliación trabajo-familia, la prevención de riesgos o la inclusión y no discriminación laboral, por nombrar algunos ejemplos.

En la primera parte, el artículo describe los aspectos más importantes de la RSI desarrollados en la literatura especializada, así como expone un resumen de algunos estudios e investigaciones sobre la importancia de las condiciones laborales de los docentes para alcanzar una educación de calidad.

En la segunda parte se exponen los principales resultados y conclusiones del estudio de caso desarrollado mediante metodología de investigación cualitativa, con el fin de analizar la aplicación de la responsabilidad social en el ámbito interno de su establecimiento educacional desde la perspectiva de los docentes de aula.

Se concluye que en el establecimiento analizado los principales aspectos de la RSI planteados a nivel teórico no están presentes o no se encuentran adecuadamente desarrollados.

\section{Revisión de la literatura}

\subsection{El ámbito interno en el modelo de la responsabilidad social}

La responsabilidad social relacionada con el ámbito interno de las organizaciones se transforma en una línea de trabajo muy interesante y reciente, para plasmar estrategias y acciones que configuren y consoliden el comportamiento socialmente responsable de una organización (Ngoc, 2012; Peláez, 2014; Mei, 2015). Así, un aspecto especialmente relevante se concentra en los esfuerzos por conectar la gestión de recursos humanos de la organización con las estrategias de RSI (Gaete, 2010; Dupont et al., 2013; Patrus et al., 2013; Saldarriaga, 2013; Berber, 2014; Mei, 2016).

Precisamente, los aspectos relacionados con la RSI se posicionan en la línea de trabajo que han señalado durante las últimas décadas organismos internacionales tales como la Organización Internacional del Trabajo (OIT) o la Organización de Naciones Unidas (ONU) en cuanto al trato y condiciones laborales proporcionados a los trabajadores por parte de las organizaciones laborales en general.

De tal modo, el comportamiento socialmente responsable de las organizaciones -entendido como la preocupación por los impactos generados en los intereses y necesidades de las partes implicadas y la sociedad en su conjunto- necesariamente debe enfocar un aspecto 
importante de su aplicación en el ámbito interno de cada institución, específicamente respecto de las personas que desempeñan un puesto de trabajo.

Según Muñoz (2007, p.113) la RSI “se ocupa de la gestión social y solidaria de los recursos humanos de las empresas y, por tanto, es la parte de la responsabilidad social corporativa que estudia el diseño e implantación de planes de igualdad y conciliación en las mismas". Asimismo destaca el importante rol de la mujer como un factor emergente en cuanto a la aplicación de la responsabilidad social al interior de las organizaciones, identificando las siguientes cuestiones relevantes en el diseño de dichos planes de igualdad y conciliación:

- Protección de la maternidad

- Flexibilidad horaria

- Teletrabajo

- Jornada a tiempo parcial y jornada reducida

- Ayuda a la familia del empleado

- Igualdad de oportunidades

- Prevención y tratamiento del acoso laboral y de la violencia de género

- Cultura de la conciliación e igualdad

El propio Libro Verde de la Unión Europea (2001, p.8) precisa el ámbito de la dimensión interna de la responsabilidad social y señala que "las prácticas responsables en lo social afectan en primer lugar a los trabajadores y se refieren a cuestiones como la inversión en recursos humanos, la salud y la seguridad y la gestión del cambio", identificando claramente dónde y respecto de quiénes debe desarrollarse el comportamiento socialmente responsable al interior de las organizaciones laborales.

De tal forma, entre los desafíos emergentes para la gestión de recursos humanos en un contexto de RSI es posible mencionar los siguientes: gestión de la diversidad, desarrollo de ambientes laborales saludables, conciliación de la vida familiar y laboral, políticas de igualdad, beneficios sociales, ética, relación con los sindicatos, seguridad e higiene, igualdad de oportunidades, gestión del acoso moral en el trabajo y diseño de puestos de trabajo, entre otros aspectos (García y Duque, 2012; Duque et al., 2014; Mory et al., 2015; Mei, 2016; Ngoc \& Fassin, 2016).

Según García y Duque (2012), cuando se analiza la interacción entre gestión humana y responsabilidad social empresarial es posible identificar algunas temáticas que permiten concretar estrategias específicas para gestionar la RSI tales como incorporación y adaptación de las personas, compensación, bienestar y salud de las personas, desarrollo del personal y relaciones con el empleado.

Finalmente, según Curto (2012) existen cuatro dimensiones de importancia a la hora de desarrollar políticas de responsabilidad social en el ámbito interno de las organizaciones presentes en la siguiente tabla. 
Tabla 1. Dimensiones de una política de responsabilidad social interna.

\begin{tabular}{|c|l|}
\hline Dimensión & \multicolumn{1}{c|}{ Aspectos principales } \\
\hline \multirow{2}{*}{ Organizativa } & Adaptación y gestión del cambio \\
\cline { 2 - 3 } & $\begin{array}{l}\text { Diálogo social (con los representantes sindicales) en las relaciones } \\
\text { laborales }\end{array}$ \\
\hline \multirow{2}{*}{$\begin{array}{c}\text { De las personas } \\
\text { (trabajadores) }\end{array}$} & Políticas de formación y aprendizaje \\
\cline { 2 - 2 } De las relaciones & Políticas de igualdad, no discriminación e integración de discapacitados \\
\hline & $\begin{array}{l}\text { Participación, implicación y compromiso de los empleados en los } \\
\text { beneficiosy el capital de la empresa, en su gestión y en el voluntariado } \\
\text { corporativo }\end{array}$ \\
\cline { 2 - 3 } & $\begin{array}{l}\text { Eliminación de riesgos laborales a través de mayor seguridad e } \\
\text { higiene en el puesto de trabajo }\end{array}$ \\
\hline Ambiental & Conciliación de la vida familiar y laboral \\
\hline
\end{tabular}

Fuente: Elaboración propia, basada en Curto (2012).

En la Tabla 1 se ratifica una parte importante de los ámbitos, orientaciones y principales formas de expresión de la responsabilidad social aplicada al interior de una organización enunciados hasta ahora, transformando al área de gestión de recursos humanos en el principal soporte interno para desplegar las políticas y acciones enmarcadas en el concepto de RSI, facilitando así el tránsito desde una gestión de recursos humanos habitualmente más concentrada en aspectos procedimentales (Gaete, 2010; García y Duque, 2012; Dupont et al., 2013; Lapina et al., 2014; Díaz y Lima, 2015; Mei, 2015).

Actualmente, la Norma SA 8000 de Responsabilidad Social creada en 1997 es la que otorga mayor atención al comportamiento socialmente responsable de las organizaciones en el ámbito interno respecto de sus recursos humanos.

Se trata de una norma uniforme y auditable ya que es aplicada por terceros actores. La misma permite obtener una certificación por tres años, enfocándose principalmente en aspectos internos del concepto de responsabilidad social, especialmente en lo referido al mejoramiento de la situación laboral y a las malas condiciones de trabajo, eliminación de las discriminaciones y la desigualdad al interior de las empresas y el fomento del desarrollo integral de las personas en la organización.

\subsection{Condiciones de trabajo de los docentes}

En la literatura especializada existen diferentes estudios e investigaciones sobre las condiciones de trabajo de los docentes a nivel mundial que evidencian los problemas y carencias con los cuales este tipo de profesionales deben desarrollar su labor. Las principales conclusiones de la gran mayoría de dichas indagaciones están relacionadas con la capacidad de contratar y retener a los buenos profesores (Barber y Mourshed, 2008) y con la mejora de las condiciones de trabajo y las estructuras salariales de los profesores (Ávalos et al., 2010; Cabezas y Claro, 2011; Medeiros, 2012), lo que se transforma en aspectos claves para elevar la calidad de cualquier sistema de educación. 
Respecto de lo anterior, diferentes organismos internacionales destacan la importancia de las condiciones laborales en las cuales los docentes desarrollan su quehacer. Así, por ejemplo, la Organización para la Cooperación y el Desarrollo (OCDE) (2009:192) señala que "para que los sistemas escolares garanticen que haya docentes de calidad, no sólo necesitarán atraer a personas capaces a la profesión docente, sino que también requerirían conservar y formar más a los docentes actualmente empleados en las escuelas". Adquieren así una gran relevancia aquellas estrategias de gestión de recursos humanos que permitan retener a los profesores en las escuelas, tales como salarios competitivos, buenas condiciones de trabajo, satisfacción en el empleo y oportunidades de formación, entre otras.

Más recientemente, pero en la misma línea de lo planteado por la OCDE, según el estudio de Bruns y Luque (2014) investigadores del Banco Mundial ratifican que la calidad de la educación está condicionada por la calidad de los profesores (Barber y Mourshed, 2008; Cabezas y Claro, 2011). En este contexto resulta imprescindible ejecutar eficientemente los procesos clave de gestión de recursos humanos tales como reclutar, desarrollar y motivar a mejores profesores.

\section{Tabla 2. Procesos clave de gestión de recursos humanos para retener a los profesores de calidad.}

\begin{tabular}{|c|c|c|}
\hline Reclutamiento & Desarrollo & Motivación \\
\hline $\begin{array}{l}\text { Incrementar la selectividad } \\
\text { elevando los parámetros } \\
\text { para el ingreso a los } \\
\text { programas de educación } \\
\text { docente; elevar la calidad de } \\
\text { los institutos de educación } \\
\text { docente; elevar los } \\
\text { parámetros de contratación } \\
\text { de los nuevos profesores. }\end{array}$ & $\begin{array}{l}\text { - Inducción: durante los } \\
\text { cinco primeros años de } \\
\text { contratación. } \\
\text { - Evaluación periódica de las } \\
\text { fortalezas y debilidades de } \\
\text { cada profesor. } \\
\text { - Desarrollo profesional: } \\
\text { capacitación para subsanar } \\
\text { debilidades y potenciar } \\
\text { capacidades. } \\
\text { - Gestión: correspondencia } \\
\text { entre asignación de } \\
\text { profesores y necesidades de } \\
\text { las escuelas. }\end{array}$ & $\begin{array}{l}\text { - Recompensas profesionales } \\
\text { tales como satisfacción } \\
\text { intrínseca, reconocimiento } \\
\text { y prestigio, crecimiento } \\
\text { profesional, dominio } \\
\text { intelectual y condiciones de } \\
\text { trabajo agradables. } \\
\text { - Presión por rendir cuentas, } \\
\text { estabilidad laboral, } \\
\text { retroalimentación de los } \\
\text { clientes, retroalimentación } \\
\text { de los directivos. } \\
\text { - Incentivos financieros: } \\
\text { pago de bonificaciones, } \\
\text { jubilaciones y beneficios, } \\
\text { diferencias salariales. }\end{array}$ \\
\hline
\end{tabular}

Fuente: Bruns y Luque (2014).

En la tabla 2 es posible observar algunos ejemplos de las acciones y estrategias que las escuelas exitosas implementan para alcanzar un mayor nivel de calidad de la educación, a través de la incorporación y retención de profesores excelentes. A tal fin es necesario que los directivos destinen recursos y esfuerzos institucionales para fortalecer los procesos clave para gestionar eficientemente a su capital humano.

Respecto de la realidad chilena también existen algunos estudios sobre las condiciones de trabajo de los docentes, como por ejemplo el desarrollado por Cornejo (2009) con docentes de enseñanza media de la ciudad de Santiago en Chile. En dicha investigación se plantea un Índice General de Bienestar (IGB) que busca analizar el bienestar/malestar de los docentes con su trabajo considerando aspectos tales como agotamiento emocional, satisfacción vocacional, condiciones materiales de trabajo (remuneraciones, jornada de trabajo), condiciones psicosociales de trabajo (control, apoyo directivo, cantidad de 
alumnos) y variables personales. En su análisis, Cornejo (2009) concluye que en la muestra de docentes considerados en el estudio, los aspectos emocionales relativos al burnout muestran niveles muy altos, por encima de la media nacional.

Asimismo, Cabezas y Claro (2011) analizan la valoración social del docente en Chile, identificando algunos factores que generan una baja valoración: remuneraciones de la carrera docente, incentivos salariales, calidad de la formación inicial, evaluación del desempeño docente y la extensa jornada laboral.

Finalmente, Eyzaguirre (2015) señala que la profesión docente es una de las peor remuneradas en el contexto de las profesiones universitarias en Chile (Cabezas y Claro, 2011). Se encuentra también por debajo en la comparación con los docentes de los países de la OCDE. A juicio de este autor, las remuneraciones de los docentes debe ser uno de los aspectos centrales a incluir en el nuevo proyecto de Ley de Carrera Docente en nuestro país por tratarse de un aspecto clave para mejorar los niveles de calidad en la educación, tal como muestra la evidencia internacional al respecto.

A partir de la revisión de la literatura es posible afirmar que la RSI puede transformarse en un interesante espacio de posibilidades que ayuden a desarrollar y consolidar mejores condiciones laborales para los docentes a través de una gestión de recursos humanos más socialmente responsable en cada escuela o unidad educativa.

\section{Metodología}

La investigación se desarrolla siguiendo un enfoque cualitativo debido al interés en el análisis de la problemática objeto de estudio desde una perspectiva interpretativohermenéutica a través de las experiencias y puntos de vista de los docentes de aula respecto de las condiciones y características laborales relacionadas con su trabajo como profesores. El objetivo principal de la investigación es comprender el desarrollo y repercusiones de las acciones de responsabilidad social interna en el Liceo Científico Humanista "La Chimba" B-36 de la ciudad de Antofagasta en Chile, como estudio de caso único e intrínseco (Stake, 2007). Las unidades de análisis fueron elegidas a través de una selección intencionada de los sujetos que cumplen con las siguientes características:
a. Ser docentes de Aula de Educación Básica y Media del Liceo Científico Humanista "La Chimba" B-36.
b. Disponer del tiempo e interés necesarios para participar en las entrevistas.
c. Ser docentes con más de dos años de experiencia en el ámbito educacional.

Es relevante destacar que el Liceo Científico Humanista "La Chimba" B-36 pertenece al sistema de educación pública de Chile integrado por establecimientos de carácter municipal. Según Davis (2012), este tipo de instituciones se transforma en uno de los principales proveedores de educación del país y habitualmente presenta problemas de financiamiento debido al alto costo que implica la educación de estudiantes de bajos recursos.

De acuerdo con el Ministerio de Educación (2015), en Chile existen 5279 establecimientos educacionales pertenecientes al sistema municipal, de un total de 16034 establecimientos públicos y privados incluyendo educación parvularia, básica y media. A nivel municipal, en Chile existen 649 establecimientos del tipo científico-humanista para jóvenes de educación media o secundaria. 
La técnica de recolección de la información es la entrevista semiestructurada. Para su desarrollo se utiliza un guión de tópicos relevantes para la investigación, y el criterio orientador para la realización de las entrevistas es el principio de saturación teórica. En total se realizaron ocho entrevistas a docentes de aula durante el segundo semestre del año 2014.

En cuanto al análisis de la información se utiliza el procedimiento establecido por la teoría fundamentada en los datos y se identifican categorías, conceptos y códigos que emergen de las entrevistas según lo señalado en la siguiente tabla.

\section{Tabla 3. Categorías de análisis de los datos.}

\begin{tabular}{|c|c|c|c|}
\hline Códigos & Conceptos & Categorías & Categoría central \\
\hline Rupturas matrimoniales & \multirow{3}{*}{$\begin{array}{l}\text { Conciliación } \\
\text { trabajo y familia }\end{array}$} & \multirow{3}{*}{$\begin{array}{l}\text { Calidad de vida } \\
\text { laboral }\end{array}$} & \multirow{8}{*}{$\begin{array}{l}\text { Responsabilidad } \\
\text { social interna (RSI) }\end{array}$} \\
\hline Jornada laboral & & & \\
\hline Rol parental de los docentes & & & \\
\hline Protocolos de evacuación & \multirow{2}{*}{$\begin{array}{l}\text { Prevención de } \\
\text { riesgos }\end{array}$} & \multirow{5}{*}{$\begin{array}{l}\text { Condiciones de } \\
\text { trabajo }\end{array}$} & \\
\hline Accidentabilidad & & & \\
\hline Nivel salarial & \multirow{3}{*}{$\begin{array}{l}\text { Remuneración e } \\
\text { incentivos }\end{array}$} & & \\
\hline Reconocimiento & & & \\
\hline Bonos & & & \\
\hline
\end{tabular}

Fuente: elaboración propia

Según la literatura expuesta anteriormente, las categorías de análisis de la RSI consideradas en nuestra investigación y presentes en la tabla 3 son la calidad de vida laboral y las condiciones de trabajo de los docentes de aula. Ambas categorías fueron señaladas por los entrevistados de nuestro estudio como aspectos que afectan significativamente su labor docente.

\section{Resultados}

\subsection{Conciliación trabajo y familia}

Desde el punto de vista de la RSI la conciliación trabajo y familia es un ámbito muy relevante. Sin embargo, en el caso estudiado no se ha tomado en consideración pues los docentes no cuentan con la suficiente cantidad de horas para estar con sus familias debido a la extensa jornada laboral que supera las nueve horas dentro del establecimiento en la realización de las tareas propias del quehacer educacional (Cornejo, 2009; Cabezas y Claro, 2011; Medeiros, 2012).

Algunos entrevistados señalan que depende mucho de la comprensión de la familia o la pareja, quienes les acompañan en el proceso e incluso les ayudan en su quehacer educativo:

“Me favorece que mi pareja o esposa me apoya mucho, en algunas ocasiones me ayuda a corregir pruebas, entonces en eso compartimos un tiempo juntos [...] es decir, me apoya, creo que es una circunstancia personal y no es lo mismo que les sucede a otros colegas que terminan con una familia separada o situaciones que llegan al tema de salud". (Entrevistado $N^{\circ} 1$ ) 
“La labor que se realiza dentro del establecimiento educacional es ardua y no deja tiempo para la familia y, es más, en ocasiones muchos de los que trabajamos en la educación nos llevamos parte del trabajo a la casa, por lo tanto, la labor docente demanda mucho más tiempo". (Entrevistado $N^{\circ} 6$ )

Una de las consecuencias de no poseer medidas para conciliar lo laboral y lo familiar se relaciona con la estabilidad del matrimonio de algunos docentes entrevistados, debido a la exigencia horaria y al trabajo que los docentes llevan a su hogar:

“Estuve casado y me separé por lo mismo, veía muy propenso eso, trabajaba ella y trabajaba yo, de ocho a ocho o nueve, depende del horario del otro colegio, y lamentablemente la veía en la semana 4 días, y los cuatro días tres horas, ¿se puede conciliar? Sí, pero yo no pude conciliar... bueno en la parte familiar con mi esposa... perdón... ex esposa, ahora con mi familia, vivo con mis padres, gracias a Dios ya aprendí de mi error, tengo las mismas o menos horas, pero llega la instancia de fin de semana digo: 'mamá, hermana vamos a comer afuera o relajarnos, a la playa o donde sea'. El fin de semana no se toca, ni siquiera para fútbol o clases particulares, entonces durante la semana no hay conciliación entre el trabajo y la familia". (Entrevistado N²)

"El costo de esto es descuidar la vida matrimonial, social y familiar. Esa falta de atención hacia ellos también provocará otras situaciones adversas y algunas veces irreversibles. Tengo una frase que plantea mi respuesta: 'TRABAJO PARA VIVIR, NO VIVO PARA TRABAJAR'. Ese es mi lema, por esto no resuelvo lo pendiente en el hogar, trato de realizar mis actividades como docente en el establecimiento aunque cuesta bastante porque cuidamos cursos o realizamos otras actividades que van en contra del aprovechamiento del tiempo". (Entrevistado $\mathrm{N}^{\circ} 4$ )

El trabajar en educación significa para los docentes un compromiso total que puede abarcar ámbitos económicos, emocionales y afectivos. Este último aspecto es el que más se desarrolla dentro de la institución educacional estudiada dado que los estudiantes carecen de afecto y atención en sus hogares. Esto se debe a que el sistema de educación municipal acoge a los sectores más pobres y con necesidades sociales específicas (Davis, 2012). Por ello, quienes deben suplir este rol son los docentes de aula, quienes deben ayudar y aconsejar en muchos aspectos académicos y afectivos a sus estudiantes.

Asimismo, en el caso estudiado no existe lugar para la conciliación debido a que la labor docente excede muchas veces las horas con las que cuentan los profesores para realizar su trabajo, por eso constantemente deben trabajar en el hogar (Cabezas y Claro, 2011). Lo anterior tiene como consecuencia el hecho de que los docentes no disponen de mucho tiempo para compartir con sus familias.

Para los docentes, la principal causa de esta problemática radica en las largas reuniones o consejos de profesores que se realizan semanalmente, la revisión de pruebas, el análisis y la realización de evaluaciones. Las actividades extracurriculares deben ser planificadas en el hogar, por ende la jornada laboral se extiende y puede llegar a ser de doce a catorce horas diarias (Medeiros, 2012), lo que perjudica el vinculo familiar o de pareja.

Hay muchos docentes que manejan los tiempos dentro del establecimiento para realizar todas las actividades en el lugar de trabajo. Aun así el tiempo es acotado y no se alcanza a realizar todas las tareas a pesar de las horas que se está en la institución. Así, el poco tiempo con que cuentan fuera del lugar de trabajo lo ocupan en hacer lo que les faltó, por lo tanto el tiempo es relativamente poco para establecer lazos o estar con la familia: 
"Yo manejo los tiempos, en mis horarios de planificación reviso evaluaciones y confecciono las planificaciones, etc., y si me queda un poco de tiempo lo aprovecho para hacer pruebas, para no llevar el trabajo a la casa, porque eso genera un problema, que nosotros llevamos a nuestras familias y ahí se generan conflictos intrafamiliares, por ejemplo, los profesores y su familia". (Entrevistado N³)

"Lo que empeora también socializar con la familia es la cantidad de trabajo que debemos llevarnos para la casa, porque el tiempo en el liceo se hace muy corto, delegando deberes para la casa. Eso a mí me ha traído tremendas complicaciones porque al tener un hijo no le puedo dedicar un tiempo de diversión, sino de estudiar, cumplir con los deberes, materiales, etc. Ponte tú, salimos a las 18:30... en llegar a la casa, en tener que ver las cosas de la casa, estudio, revisión de pruebas, planificar y buscar material para las clases... ¿En qué momento se conversa? ¿Se habla de cosas que pasan en la vida cotidiana? Casi nunca". (Entrevistado N5)

Conciliar la vida de padres e hijos es un proceso complejo porque lleva años acostumbrarse a los horarios de uno u otro, tomando en cuenta que la educación no solo la realiza el colegio o escuela sino también los padres, quienes deben inculcar valores a sus hijos. Sin embargo, los docentes plantean que una vez que finaliza la jornada laboral llegan a sus casas a realizar las mismas tareas que hicieron durante el día, es decir, ayudar y orientar la educación de sus hijos, que es una tarea más desgastante y que requiere mucho más tiempo para reforzar los aspectos valóricos y académicos de sus hijos.

De esta manera, los hijos de los profesores muchas veces son los más perjudicados por la vida laboral de sus padres. La conciliación puede lograrse siempre y cuando existan las instancias necesarias para que los padres puedan cumplir con su rol en el hogar. Lamentablemente, en la actualidad no existe ninguna regla o medida que se adopte para mejorar la relación entre padres e hijos:

“Lleva años para poder lograrlo, porque es muy difícil. Yo soy una persona súper responsable, yo tengo que estar en la casa a cierta hora, debo cumplir con las tareas de mi hijo, de ser mamá, llegar a casa y realizar las mismas tareas que hago todo el día, hacer tareas, porque en el colegio que va mi hijo tiene jornada parcial, solo una jornada, entonces un $50 \%$ de la educación la debe realizar el apoderado en la casa y, también es un colegio municipal y tiene más de 10 años de excelencia académica, entonces es un colegio que te exige y mucho. Por ejemplo: un niño de segundo básico ya sabe dividir, ellos tienen muy buen colegio y el esfuerzo que te piden como mamá es al máximo total, entonces una vez que termino mi jornada laboral, llego a casa a trabajar con mi hijo". (Entrevistado $\mathrm{N}^{\circ} 7$ )

La mayoría de las veces la vida familiar es la más perjudicada debido al trabajo que realizan los docentes de aula diariamente. A tal fin la RSI plantea que pueden emplearse medidas para revertir esta situación, pero a nivel directivo no están familiarizados o desconocen términos tales como el teletrabajo, que consiste en disminuir las horas de planificación en el establecimiento y desarrollarlas en el hogar, cerca de la familia. Esto tiene como consecuencia que el trabajador realice sus labores de una forma más eficaz y eficiente, aprovechando los tiempos que son asignados para la labor pedagógica. Otra opción es manejar los horarios de ingreso y egreso del establecimiento educacional. 
Para conciliar trabajo y familia se necesita un entorno laboral y familiar que permita la reciprocidad entre el trabajador y empleador, lo que se convierte en un valor compartido para la sociedad y la institución. Esto promueve el desarrollo de salud mental y seguridad del trabajador (Cornejo, 2009), trayendo consigo motivación y estabilidad dentro de la institución.

Asimismo, la RSI insta a las instituciones a establecer nuevos patrones o instancias en que las que se pueda conciliar el aspecto laboral y familiar. Uno de ellos es el teletrabajo que, gracias a la tecnología, puede implementarse sin problema alguno y que disminuye la carga de trabajo de los docentes, especialmente de hombres y mujeres que comparten roles dentro del hogar.

\subsection{Prevención de riesgos}

La RSI establece que las instituciones deben preocuparse por la seguridad y salud de sus trabajadores, previniendo cualquier tipo de accidentes físicos, químicos, laborales o biológicos. Cada empleador debe abonar un seguro a todos sus trabajadores en caso de sufrir algún percance o daño a su integridad física o psicológica. Asimismo, las instituciones deben considerar las enfermedades profesionales que aquejan a los docentes del sistema municipal.

Con respecto a lo anterior, en el establecimiento educacional analizado no existen reglamentos para actuar en caso de emergencias tales como sismos, incendios, tsunamis, entre otros. Se desconocen los protocolos a seguir en momentos determinados, y no se ha proporcionado a los docentes de aula cursos sobre manejo de extintores en caso de incendio, pues para evitar el riesgo de un uso indebido debe saberse cómo funcionan y cómo utilizarlos.

“Con los riesgos como incendios y terremotos, en el caso de los extintores, falta la parte práctica de utilizarlos. En cuanto a incendios o lo que sea, hemos tenido poco ensayo y práctica, a lo mejor yo sé usar extintor pero no todos lo saben, entonces es necesario un pequeño curso de IST". (Entrevistado N³)

En caso de sismos existen dentro de la institución zonas establecidas para la evacuación. Sin embargo, las indicaciones fueron realizadas con cintas que con el paso del tiempo se van deteriorando, por lo que no cumplen con la función para las que se utilizan. Asimismo, los docentes de aula plantean que las zonas de seguridad no están en las cotas de seguridad en la Avenida Pedro Aguirre Cerda, lugar que las autoridades de nuestra ciudad han establecido como zona segura en caso de sismos que generen como consecuencia un tsunami.

"No sabemos en qué parada estamos, por el tema reciente con los dos terremotos. Recién hicimos un simulacro u operativo Deyse ${ }^{1}$ para analizar en qué situación real estamos, y ahora pasó lo del terremoto y aún estamos esperando y dijeron que iban a realizar un nuevo simulacro sorpresa. No nos han dicho nada". (Entrevistado N³)

Actualmente no tienen lugar simulacros periódicos para dar a conocer los procedimientos o formas de evacuación para llegar a los puntos de encuentro de seguridad que se hallan demarcados en el sector norte de la ciudad. 
Cabe recalcar además que en el lugar donde se encuentra el Liceo Científico "La Chimba" hay otros dos establecimientos educacionales más, por lo tanto es necesario conocer cuáles son los parámetros o protocolos a seguir durante alguna emergencia. Se debe asimismo manejar la variable de que estos dos establecimientos poseen un protocolo establecido y que es diferente al del Liceo "La Chimba".

La RSI es clara acerca de la gestión de la seguridad de la institución. Existen profesionales destinados a mejorar la prevención de riesgos en la institución. Se plantea la necesidad de crear un comité de seguridad que dicte las reglas de comportamiento ante diferentes situaciones en las que se ponga en riesgo la vida de los docentes de aula del establecimiento.

“Verdad es que es un tema que me preocupa muchísimo, porque no tenemos un protocolo adecuado para realizar en caso de diversas amenazas de peligro, desde balazos, una bomba o un maremoto. Solo sabemos que debemos evacuar el establecimiento. Aún no está conformado un comité con el que se pueda contar en caso de emergencias. Hay un protocolo de evacuación pero nada más dentro del liceo". (Entrevistado №4)

“Dentro de los pocos elementos que existen para la prevención de riesgos se encuentra la marcación de las salidas de emergencia en todos los pasillos y pisos del establecimiento. Además de los ensayos (pocos) de evacuación y los pasillos y escaleras designados para cada profesor". (Entrevistado $\mathrm{N}^{\circ} 8$ )

En el establecimiento no existe ningún comité paritario o de seguridad, el cual por lo general se encarga de establecer las reglas o escribir los protocolos a seguir en caso de un imprevisto en el establecimiento. Tampoco existe la implementación de seguridad necesaria en caso de accidente laboral de un docente. Los profesores, auxiliares y asistentes de la educación no cuentan con los conocimientos mínimos de primeros auxilios. Excepto los docentes de Educación Física y Biología, los demás no han sido ni están capacitados y no sabrían cómo actuar frente a una situación de riesgo o accidente.

“No hay implementación adecuada en caso de un accidente laboral. No existe ni una capacitación para atender en una emergencia, que en este caso como docentes deberíamos tener algún curso de primeros auxilios mínimos". (Entrevistado Nº6)

Actualmente el establecimiento cuenta con una enfermería en caso de accidente. La misma cuenta con los elementos básicos para resolver situaciones de emergencia como un corte o herida simple, pero en caso de un corte profundo el docente o estudiante debe ser traslado de inmediato a un consultorio u hospital más cercano para su asistencia. Lo mismo sucede en caso de lesión ósea o esguince. Esta sala de enfermería no cuenta con los elementos necesarios para una lesión expuesta y ante esto debe trasladarse inmediatamente al lesionado al Centro Hospitalario del Norte, el centro asistencial médico más cercano al establecimiento.

“Pero en cuanto a todo lo que tiene que ver en sí con accidentes escolares, el otro día se cayó una niña. ¿Por qué? ¿No se les dice a los auxiliares que no deben trapear cinco minutos antes de que los niños salgan al recreo, que no pueden trapear los baños cuando cinco minutos después tocan el timbre para el recreo? O sea, ¿de qué estamos hablando? A lo mejor les falta pedir una charla, a alguna institución, en nuestro caso a la $\mathrm{ACHS}^{2}$, en donde se capacite a todas estas personas sobre cómo deben hacer su trabajo. Yo no he ido a la cocina a ver cómo están trabajando, te apuesto que no usan guantes ni mascarillas, pero eso lo tiene que realizar el Comité Paritario, no puede hacer mucho el Comité de Seguridad". (Entrevistado $N^{\circ} 7$ ) 
La RSI plantea que las instituciones deben promover la prevención de riesgos en los lugares de trabajo, contribuyendo así a la convivencia sana dentro de la institución, aumentando la productividad. En el caso de este liceo disminuiría el ausentismo laboral y fortalecería las habilidades y capacidades de los docentes dentro del establecimiento y del aula. La institución debe mejorar las condiciones laborales de los trabajadores, principalmente en el ámbito estructural, para que los docentes y no docentes puedan desarrollar de mejor manera sus labores diariamente.

\subsection{Remuneraciones e incentivos}

Según los estudios, las bajas remuneraciones y escasos incentivos económicos se transforman en otro aspecto clave dentro de las condiciones de trabajo que deben mejorarse en el caso de los docentes chilenos (Valenzuela et al., 2010; Cabezas y Claro, 2011; Medeiros, 2012; Eyzaguirre, 2015), situación respecto de la cual el presente estudio tampoco queda indiferente.

Los salarios de los docentes de aula del Liceo Científico Humanista "La Chimba" son muy bajos, especialmente en el contexto de la realidad socioeconómica de la ciudad de Antofagasta ${ }^{3}$, que posee uno de los índices más altos en cuanto al costo de vida, sobre todo los valores de casa y/o víveres. Para un docente con cuarenta y cuatro horas de trabajo semanales, con hijos o una familia conformada por cuatro personas, el sueldo no está acorde a las necesidades o el valor de los artículos de primera necesidad, los que son altísimos comparados con los de otras regiones centrales del país.

"El sueldo de un profesor no es un sueldo digno, no es algo que digamos nosotros sino que lo observa la sociedad en general, nuestra sociedad chilena. Cuando un joven se proyecta estudiar pedagogía vienen los problemas y el cuestionamiento de la familia, que les plantea el hecho de que no será una carrera con estabilidad económica, que pasarán penurias". (Entrevistado $\mathrm{N}^{\circ} 3$ )

"Considero que es bastante poco. En contraste con las horas de trabajo que uno entrega en esta labor como profesor, las horas de reuniones de apoderados y atención de apoderados no son consideradas al momento de recibir el sueldo, además no son lo suficientemente remuneradas, no se contempla esto como parte del trabajo en las remuneraciones". (Entrevistado $\mathrm{N}^{\circ} 6$ )

Las malas remuneraciones que existen a nivel municipal han traído diversas consecuencias, pero la principal es la alta rotación de personal que existe en los establecimientos educacionales municipales (Valenzuela et al., 2010; Cabezas et al., 2011). Debido a las bajas remuneraciones existentes los docentes cambian de trabajo muchas veces por necesidad. El salario no es suficiente para mantener al grupo familiar, por lo tanto debe emigrar a un establecimiento particular o particular subvencionado para poder solventar los gastos de una familia.

"Yo trabajaba en un colegio particular en el cual ganaba un ochenta por ciento más de lo que gano ahora, con menos horas, pero la exigencia era mayor, la exigencia me encanta, no me refiero al papeleo". (Entrevistado $\mathrm{N}^{\circ} 2$ )

Otro factor relevante para los docentes de aula es el detalle de las remuneraciones, las que están bien especificadas en la liquidación de sueldo pero nunca es igual para los docentes aunque tengan la misma cantidad de horas, mismos años de servicio y título profesional (Cabezas y Claro, 2011). Del tal modo, existen asignaciones o complementos dentro de 
la remuneración que varían en su valor constantemente sin notificación a los docentes, por ejemplo las asignaciones de Bono Proporcional de Educación o Bono de Excelencia Académica, las que varían según la cantidad de años de servicio del docente y la cantidad de horas dentro del establecimiento educacional. Asimismo, el Bono de Reconocimiento Profesional no se abona con regularidad y los docentes deben esperar al menos tres meses para recibir este pago.

“El pago de la remuneración es bastante complejo y engorroso en un principio, nunca pero nunca se gana lo mismo, la renta es variable y la mayoría de las veces falta dinero en la remuneración, los bonos hay que ir a pelearlos a la Corporación". (Entrevistado N6)

Los docentes de aula no solo se sienten motivados por los incentivos monetarios, sino también por aquellas situaciones de reconocimiento por la labor realizada durante su jornada laboral.

Lamentablemente, en la actualidad no existen actos de empatía entre los docentes y directivos, principalmente con la dirección del establecimiento; no existen palabras de aliento ante las buenas acciones pedagógicas realizadas por los docentes, no hay reconocimiento al trabajo realizado e incluso en vez de ayudar a la buena convivencia dentro del establecimiento generan lo opuesto. A su vez, los docentes sienten que se les cierran las puertas al momento de crear instancias que mejoren el aprendizaje de los estudiantes y que no les es posible realizar estos cambios para los estudiantes del plantel educacional.

“No me siento motivado por la plana directiva para hacer cosas nuevas, al saber cosas las puedo implementar, pero no me siento motivado para hacerlo, no me refiero a plata, no me muevo si me la gasto y listo, pero te insisto no me mueve, me encanta que me digan: 'Profe, hizo bien esto'”. (Entrevistado N²)

En la actualidad no existen refuerzos positivos hacia los docentes y su labor diaria, solo se evidencian situaciones de conflicto entre dirección y docentes y no hay instancias de acercamiento entre ambos estamentos, lo que ha perjudicado el desarrollo positivo de los estudiantes y la comunidad en general. A esto se debe agregar la poca o nula empatía de algunos directivos que no valoran los esfuerzos realizados por los docentes por mejorar en su quehacer pedagógico. Al contrario, siempre hay críticas negativas hacia su labor:

“Acá no hay ninguno, para mí ningún incentivo que me diga: 'Profesora, como usted fue a una reunión de salud y estuvo dos horas fuera de su horario, le vamos a compensar y hoy entre más tarde o váyase más temprano', o sea, si no 'pesco' mi bolso y me voy". (Entrevistado $\mathrm{N}^{\circ} 7$ )

Tal como se analizara en los párrafos anteriores, no hay buena recepción para el trabajo realizado por los docentes en el establecimiento, no existen instancias en que se valore o reflexione acerca de la buena labor que efectúan los docentes hacia los estudiantes aun cuando se dedica más del tiempo del que establece el contrato de trabajo. Asimismo, no hay recompensas escritas ni verbales hacia el buen trabajo realizado por la mayoría de los docentes del establecimiento educacional de enseñanza media.

Finalmente, en la unidad educativa estudiada durante el año 2014 los docentes de aula recibieron dos tipos de incentivos económicos adicionales por el desempeño de su labor: 
A. Bono de Excelencia Académica. Es un bono que reciben los docentes cada tres meses, en los meses de marzo, junio, septiembre y diciembre. El monto asciende a los $\$ 200.000$ (doscientos mil pesos chilenos). Es un reconocimiento a la labor de los docentes y a los resultados obtenidos en el SIMCE ${ }^{4}$ durante el año 2013, en el cual los estudiantes de segundo medio del establecimiento obtuvieron el primer lugar a nivel municipal de la región, incluso mejor que liceos emblemáticos de la ciudad como Andrés Sabella o Domingo Herrera Rivera. Este bono fue percibido durante dos años consecutivos, 2014 y 2015. Esto supone un incremento en la remuneración de los docentes y también permite un beneficio extra o incentivo para realizar su labor diariamente en el establecimiento. Sin embargo, algunos entrevistados señalan que este incentivo no debe ser cada tres meses sino todos los meses:

"Independientemente de los beneficios en bonos u otros dineros que se nos cargan al sueldo como la Excelencia Académica, que es aproximadamente un monto de \$150.000 pesos mensuales, creo y considero que es poco, contraponiendo la cantidad y calidad del trabajo que hago diaria, semanal o mensualmente". (Entrevistado $N^{\circ} 4$ )

“Reciben un bono de subvención de 200 mil pesos, suponte una o dos veces al año, en cambio nosotros recibimos 30 mil pesos al año, entonces la excelencia académica, claro que recibir 100 mil pesos cada tres meses que te van a servir cada tres meses, pero debería ser todos lo meses, porque es un trabajo que hiciste todo un año, no solo tres meses". (Entrevistado $N^{\circ} 7$ )

B. Bono de Asignación Variable por Desempeño Individual (AVDI). Depende del resultado obtenido en la evaluación docente, siempre y cuando se obtenga una evaluación competente o destacada. Sin ese logro no es posible optar a esta asignación. La crítica que se realiza a este instrumento es que nadie sabe cómo son evaluados los docentes de aula ni quiénes revisan cada uno de los aspectos medidos en una prueba en la que debe responderse un cuestionario relacionado con su asignatura. Este incentivo varía de un $10 \%$ a un $15 \%$ de la renta mensual bruta del docente de aula.

“De los que conozco se encuentra el bono de zona, el bono de reconocimiento profesional, el bono de excelencia académica y el último de asistencia completa (el cual tiene sus requisitos, no es fijo) y AVDI. Incentivos de otra índole, que no sean monetarios, no he recibido ninguno". (Entrevistado $\mathrm{N}^{\circ} 8$ )

En virtud de los testimonios de los docentes de aula entrevistados durante la investigación se constata que los niveles salariales obtenidos en el sistema de educación municipal chileno son bastante bajos en comparación con otras profesiones y es especialmente bajo en relación al poder adquisitivo en la ciudad de Antofagasta. Si bien existen algunos incentivos monetarios adicionales a la remuneración mensual, éstos se encuentran supeditados al cumplimiento de metas o niveles de desempeño que son difíciles de alcanzar debido a la elevada carga laboral de los docentes, que en la mayoría de los casos excede la jornada de trabajo por la cual están contratados. No existe reconocimiento o incentivo de carácter no monetario por parte de los directivos hacia la labor realizada por el profesorado. 


\section{Conclusiones}

A partir de la información recopilada sobre el caso estudiado es posible concluir que las acciones relativas a la RSI desarrolladas en la unidad educativa analizada son escasas, especialmente respecto de la conciliación trabajo-familia, particularmente debido a la extensa jornada de trabajo y al tiempo dedicado en su hogar a las actividades de planificación.

En base a la revisión de la literatura expuesta en el presente trabajo es posible afirmar que existen elementos que permiten reconocer una importante relación entre la gestión de recursos humanos más socialmente responsable y la generación de condiciones de trabajo adecuadas para que los docentes de aula desarrollen su labor.

Para lograr lo planteado anteriormente resultará fundamental el compromiso de los directivos y sostenedores con la implementación de políticas y estrategias de responsabilidad social interna en los establecimientos educacionales con el propósito de generar mejores condiciones de trabajo para contratar y retener a los docentes de calidad. Por ello, será altamente recomendable la realización de cursos de perfeccionamiento que fortalezcan las habilidades blandas o de empatía de los directivos para mejorar las relaciones interpersonales entre directivos y docentes y que, asimismo, les permitan implementar acciones que favorezcan la conciliación de la vida laboral y familiar de los docentes de aula de manera más efectiva, a través de la instrumentación de medidas más eficientes para generar condiciones de trabajo más adecuadas en temáticas tan importantes para los docentes como son la remuneración o la prevención de riesgos.

\section{Referencias bibliográficas}

Ávalos, B.; Cavada, P.; Pardo, M. y Sotomayor, C. (2010). La profesión docente: Temas y discusiones en la literatura internacional. Revista Estudios Pedagógicos, Vol.36, № 1, pp. 235263.

Barber, M. y Mourshed, M. (2008). Cómo hicieron los sistemas educativos con mejor desempeño del mundo para alcanzar sus objetivos. Santiago de Chile: CINDE.

Berber, N.; Stangl, G.; Slavic, A. \& Baosic, M. (2014). Relationship between Corporate Social Responsibility and Human Resource Management as New Management Concepts in Central and Eastern Europe. Engineering Economics, 25(3), pp. 360-369.

Bruns, B. y Luque, J. (2014). Profesores excelentes. Cómo mejorar el aprendizaje en América Latina y El Caribe. Washington: Banco Mundial.

Cabezas, V. y Claro, F. (2011). Valoración social del profesor en Chile: ¿Cómo atraer alumnos talentosos a estudiar pedagogía? Temas de la Agenda Pública, año 6, № 42. Santiago de Chile: Centro de Políticas Públicas UC.

Cabezas, V.; Gallego, F.; Santelices, V. y Zarhi, M. (2011). Factores correlacionales con las trayectorias laborales de docentes en Chile, con especial énfasis en sus atributos académicos. Informe proyecto FONIDE N: FS511082-2010, quinto concurso.

Comisión Europea (2001). El Libro Verde: Fomentar un marco europeo para la responsabilidad social de las empresas. Bruselas. 
Cornejo, R. (2009). Condiciones de trabajo y bienestar/malestar docente en profesores de enseñanza media de Santiago de Chile. Revista Educ. Soc., Campinas, vol 30, N 107, pp. 409-426.

Curto, M. (2012). La responsabilidad social interna de las empresas. Cuadernos de la Cátedra "La Caixa" de Responsabilidad Social de la Empresa y Gobierno Corporativo, № 16.

Davis, G. (2012). Tensiones organizacionales en la implementación de las Reformas Educacionales en el Municipio: el caso de Chile. Revista Estudios Pedagógicos, Vol. 38, № 1, pp. 311-331.

Díaz, N. y Lima, M. (2015). Responsabilidad social interna: entre la diferencia y el discurso en el escenario organizacional. Cuadernos EBAPE.BR, V. 13, № 2, pp. 346-368.

Dupont, C.; Ferauge, P. \& Giuliano, R. (2013). The impact of Corporate Social Responsibility on Human Resource Management: GDF SUEZ's Case. International Business Research, Vol. $6, N^{\circ} 12$, pp. 145-155.

Duque, J.; García, M. y Azuero, A. (2014). Responsabilidad social desde las percepciones de los trabajadores en una empresa vallecaucana del sector de clase mundial cosméticos y productos de aseo. Revista Pensamiento y Gestión, Nº 36, pp. 56-106.

Eyzaguirre, S. (2015). ¿Son atractivos los salarios de la nueva carrera docente? Puntos de Referencia, № 401. Santiago de Chile: Centro de Estudios Públicos.

Gaete, R. (2010). Discursos de gestión de recursos humanos presentes en las iniciativas y normas de responsabilidad social. Revista Gaceta Laboral, 16(1), pp. 41-62.

García, M. y Duque, J. (2012). Gestión humana y responsabilidad social empresarial: un enfoque estratégico para la vinculación de prácticas responsables a las organizaciones. Revista Libre Empresa, №17, pp. 13-37.

Lapina, I.; Maurane, G. \& Starineca, O. (2014). Human resource management models: aspects of knowledge management and corporate social responsibility. Procedia - Social and Behavioral Sciences, No 110, pp. 577-586.

Medeiros, M. (Coord.) (2012). Propuestas para una carrera docente. Santiago de Chile: Centro de Políticas Públicas UC.

Mei, P. L. (2015). The Manifestation of Internal Corporate Social Responsibility on Employee's Behaviour in Small Medium Sized Enterprises. Journal of Social Science Studies, Vol. 2, N 2, pp. 259-277.

Mei, P. L. (2016). Corporate Social Responsibility and the Evolution of Internal Corporate Social Responsibility in 21st Century. Asian Journal of Social Sciences and Management Studies, 3(1), pp. 56-74.

Ministerio de Educación (2015). Resumen estadístico de la Educación 2015. Centro de Estudios del MINEDUC.

Mory, L.; Wirtz, B. \& Göttel, V. (2015). Factors of internal corporate social responsibility and 
the effect on organizational commitment. En línea, disponible en: http://www.tandfonline. com/doi/pdf/10.1080/09585192.2015.1072103 (Visitado el 31 de enero de 2016)

Muñoz, L. (2007). Protagonismo de la mujer en la gestión de la responsabilidad social corporativa. Capital Humano: Revista para la integración y desarrollo de los recursos humanos, 20(209): pp. 112-118. En línea, disponible en: http://pdfs.wke.es/2/9/0/3/pd0000012903. pdf (Visitado 18 de julio de 2015).

Ngoc, N. (2012). Human resource training and development as facilitators of corporate social responsibility. Journal of Economics and Development Vol. 14, № 3, pp. 88-98.

Ngoc, N. \& Fassin, I. (2016). The impact of internal corporate social responsibility on organizational commitment: Evidence from Vietnamese Service Firms. International Business Management, 10(6), pp. 784-792.

OCDE (2009). Los docentes son importantes. Atraer, formary conservar a los docentes eficientes. París: OCDE.

Patrus, R.; Carvalho Neto, A.; Coelho, H. \& Teodósio, A. (2013). Corporate Social Responsibility and labor relations: a research agenda about internal stakeholders management in UN's global compact signatory corporations. Review of Business Management, 15(46), pp. 22-38. Peláez, J. (2014). La responsabilidad social empresarial y la gestión humana en Colombia: desafíos para fortalecer una relación estratégica. Revista Ciencias Estratégicas, Vol. 22, No 31, pp. 83-99.

Saldarriaga, J. (2013). Responsabilidad social y gestión de conocimiento como estrategias de gestión humana. Revista Estudios Gerenciales, № 29, pp. 110-117.

Stake, R. (2007). Investigación con estudio de casos. Madrid: Morata.

Valenzuela, J.; Sevilla, A.; Bellei, C. y De los ríos, D. (2010). Remuneraciones de los docentes en Chile: resolviendo una aparente paradoja. Serie Documentos de orientación para políticas públicas. Santiago de Chile: Centro de Investigación Avanzada en Educación de la Universidad de Chile.

(Endnotes)

${ }^{1}$ Operativo de Evacuación y Seguridad Escolar.

${ }^{2}$ Asociación Chilena de Seguridad.

3 http://www.capital.cl/negocios/2013/06/03/070654-antofagasta-o-la-nueva-dubai

${ }^{4}$ Sistema de Medición de la Calidad en la Educación. 